\title{
Epidemiología del prolapso genital
}

\author{
Dr. Joaquín Luna \\ HOSPITAL GENERAL UNIVERSITARIO DE LA SAMARITANA
}

\section{INTRODUCCION}

En la elaboración del presente trabajo hemos tenido como meta la evaluación del manejo del prolapso genital en el Servicio de Gineco-Obstetricia del Hospital General Universitario de la Samaritana.

Nuestra pretensión no es hacer una exhaustiva revisión del tema y estamos conscientes de las limitaciones del Servicio de Estadística del Hospital y de la condición de que los cirujanos son en la mayoría de las veces personal en entrenamiento, así como también nos encontramos al revisar las historias, con que la colaboración de nuestras pacientes no es la adecuada.

Definimos el prolapso genital como el descenso de las estructuras del aparato genital y/o de los elementos con él relacionados, por debajo del nivel que normalmente ocupan.

\section{NOCIONES ANATOMICAS}

El útero está suspendido elásticamente en el centro de la pelvis menor. La situación del cuerpo uterino está influida por un cordón bien delimitado, que se extiende desde los ángulos uterinos hasta la pared abdominal anterior (LIGAMENTOS REDONDOS). Este cordón elástico puede variar su longitud en relación con el aumento de tamaño del útero debido a embarazo o formaciones tumorales.

La porción cervical del útero está sujeta a la pared pelviana por encima de la vagina, por una red horizontal dispuesta en varias capas y en forma de abanico. La suspensión conjuntivo-elástica $y$ en parte muscular lisa (PARAMETRIO) está parcialmente reforzada por porciones compactas. En ambos lados se encuentran resistentes cordones fibrosos mezclados con fibras musculares lisas $y$ que se denominan ligamentos cardinales o de MACKENROTH. En el ligamento cardinal discurren los vasos uterinos por debajo del cruce de los uréteres.

El ligamento úterosacro constituye un refuerzo marginal del espacio creado en la concavidad sacra por el paso del recto.

Hacia adelante se encuentran unos fascículos conjuntivos reforzados, que constituyen los llamados ligamentos pubo-vésico-uterinos. El variable estado de repleción de la vejiga exige un espacio en la parte ventral del sistema de sustentación del cervix.

El piso de la pelvis está constituido por un potente músculo en forma de 
diafragma denominado ELEVADOR $D E L A N O$, que con sus tres porciones pubo-coccígea, pubo-rectal e ileococcígea abrazan la vagina y el recto fijándose en la línea media formando un verdadero tendón central y al cual convergen otros grupos musculares menores que tienen escasa importancia para la sustentación del piso de la pelvis.'El músculo elevador del ano rodea en varias capas la porción inferior del recto y cierra en forma de abanico el suelo de la pelvis, dejando hacia adelante un espacio triangular en el que desembocan la vagina $y$ la uretra (HIATO GENITAL). Debido a la marcha erguida y a la consiguiente presión del contenido abdominal la especie humana soporta una mayor sobrecarga sobre el piso de la pelvis que los cuadrúpedos.

\section{FISIOPATOLOGIA}

Los principales factores que pueden conducir a un prolapso genital son:

El Parto: Constituye el principal factor etiológico del prolapso. En el curso de un parto espontáneo, pero fundamentalmente en un parto operatorio (forceps, espátulas, vacuoextractor), puede producirse una elongación, distensión o desgarro de los sistemas de fijación del aparato genital (Ligamentos cardinales, útero-sacros, piso de la pelvis). Si estos desgarros no son reparados en forma adecuada o si la distensión o elongación que normalmente ocurre durante el embarazo no regresa en forma adecuada después del parto, puede llegar a producirse el prolapso.

Debilidad congénita de los medios de fijación: En un pequeño porcentaje de casos, el prolapso genital aparece en mujeres nulíparas. En estos casos se supone que la causa desencadenante fundamental es la debilidad congénita de los medios de fijación del aparato genital, con frecuencia combinada con obesidad y esteroptosis (vientre péndulo).

\section{Factores Secundarios:}

a. Edad avanzada: Favorece la producción del prolapso, ya que en esta época se produce una reducción del tamaño del útero $y$ una relajación del tono de los músculos del piso pélvico y en general de todos los medios de fijación, al disminuir el trofismo por la falla estrogénica principalmente.

b. Factores raciales: En determinadas poblaciones el prolapso es más frecuente que en otras. En la raza negra y en algunas poblaciones indias el prolapso es mucho más raro que entre la población caucásica y oriental.

c. Factor genético: Se ha señalado una predisposición genética de ciertas familias a una mayor relajación hística que favorecería la producción del prolapso.

d. Enfermedades varias: Todas las situaciones que conducen a un debilitamiento general del organismo pueden favorecer el prolapso genital, al igual que trastornos de los nervios sacros S1-S4 (espina bífida o neuropatía diabética). También otras enfermedades crónicas como asma, bronquitis crónica o bronquiectasia.

e. Hábitos: Tienen importancia también las labores realizadas por la mujer que demandan esfuerzos físicos violentos.

f. Técnicas quirúrgicas: practicadas para corregir otras patologías pueden favorecer la producción de prolapso genital. La histerectomía vaginal que no tiene en cuentá la corrección de la falla de las paredes vaginales o del enterocele existente, ni la fijación a la cúpula de los ligamentos seccionados. La histerectomía abdominal total practicada con la 
técnica extrafacial da lugar a mavor cantidad de prolapsos que la técnica subfacial o intrafacial, que conserva la integridad de la fascia pericervicovaginal en la cual se distribuyen los ligamentos transversos y úterosacros, según lo preconizan muchos grupos, entre ellos Jaszczak v Evans.

Es importante evaluar los pequeños prolapsos para corregirlos durante el curso de una histerectomía; los enteroceles en la cirugía abdominal con los puntos que cierran el Douglas por el procedimiento clásico de Moschowits, o en la vaginal disecando la pared vaginal posterior suficientemente alta para buscar el saco herniario y reforzar con la miorrafia de los elevadores, así como estrechar profilácticamente el fondo de saco posterior aproximando en su parte alta los ligamentos úterosacros y transversos, como lo preconiza Waters, citado por Bernal en su trabajo del prolapso de cúpula vaginal.

\section{CLASIFICACION DEL PROLAPSO GENITAL}

Cistocele y uretrocele: Se denomina Cistocele al descenso de la pared vaginal anterior acompañado del descenso de la pared posterior de la vejiga y del trígono. EI Uretrocele está comunmente asociado al Cistocele y a la incontinencia urinaria de esfuerzo.

Rectocele: Abombamiento de la pared vaginal posterior con inclusión parcial de la ampolla rectal. La columna fecal queda retenida fácilmente en el saco herniario vaginal.

Enterocele: También denominado prolapso del saco de Douglas, es el prolapso de la porción superior de la pared vaginal posterior, que puede contener o no asas intestinales o epiplón.
Prolapso Uterino Crónico: (Hernia del piso pélvico, hernia pudenda) es el descenso del útero por debajo del lugar que normalmente ocupa.

Prolapso Uterino Agudo: Inversión del útero en el alumbramiento y descenso por fuera de la vulva.

Prolapso de Cúpula Vaginal: Eversión o descenso de las paredes vaginales después de histerectomía.

El prolapso genital se puede clasificar en diferentes estadíos:

a. Prolapso de Primer Grado: Intravaginal o prolapso leve, en el cual las estructuras descienden una parte hacia abajo en el interior de la vagina.

b. Prolapso de Segundo Grado: (Prolapso moderado). Cualquiera que sea el origen del prolapso las estructuras descienden hasta el introito vaginal.

c. Prolapso de Tercer Grado: (Prolapso Completo). Todas las estructuras (vagina, cervix $y$ útero) sobresalen más allá del introito y la vagina se encuentra invertida, observándose por fuera de la vulva.

\section{SINTOMAS}

Los síntomas por los cuales consultan las pacientes con prolapso genital son frecuentemente:

- Sensación de peso vaginal.

- Dolor, pesadez o tirantez en el bajo vientre o en zonas lumbares.

- Trastornos urinarios consistentes en disuria polaquiuria 0 incontinencia urinaria de esfuerzo.

- Trastornos rectales como constipación o tenesmo. 
- Si el útero descendido está ulcerado, pueden existir pequeñas pérdidas hemáticas.

- Si el introito está dilatado, la vagina pronto se infecta y da lugar a la aparición de leucorrea.

\section{DIAGNOSTICO}

El diagnóstico de prolapso genital se basa principalmente en la inspección, pero siempre se debe valorar el estado muscular del piso pélvico y descartar o confirmar la incontinencia urinaria de esfuerzo y patología tumoral asociada.

\section{TRATAMIENTO}

Podríamos hablar de profilaxis del prolapso si recomendamos posteriormente al parto la práctica de ejercicios que aumenten el tono de los músculos pelviperineales, así como el esmerado manejo del parto que evite trabajos prolongados o manejos instrumentales o complicaciones previsibles; así como el empleo de técnicas quirúrgicas que eviten la lesión de las estructuras de soporte cervicovaginal, evitar las disecciones romas en la cirugía ginecológica dado que éstas favorecen la necrosis y la infección de las heridas.

El tratamiento médico propiamente dicho está bastante relegado a casos muy especiales en los cuales el estado general de la paciente no permite pensar sino en la aplicación de pesarios.

El tratamiento quirúrgico se basa en la consideración del grado de prolapso asociado o nó a elongatio colli o a incontinencia urinaria de esfuerzo o al deseo de la paciente de embarazos posteriores y relacionado todo ello a la edad. De acuerdo al análisis de estos parámetros se procede a realizar una o varias de las técnicas propuestas para la corrección como son:

- Colporrafia anterior y/o posterior en los casos de mujeres con prolapso II sintomático.

- Corrección del enterocele cuando ello lo amerite.

- Operación de Manchester Fothergil cuando en una mujer por debajo de los 40 años se asocian prolapso con elongatio colli y deseo de embarazo posterior, aunque es universalmente aceptado que la amputación del cervix reduce la fertilidad y es causa de distocia.

- Histerectomía vaginal en el caso de que el prolapso sea II o III y la edad - las condiciones de paridad así lo recomienden, siempre con el reparo de las paredes vaginales anterior y posterior y la fijación adecuada de los ligamentos uterinos.

- En los casos de prolapso II o III asociados a I.U.E. evaluamos el resultado post-operatorio en relación con ella y en caso de fracaso preferimos las técnicas de uretrocistocolpopexia retropúbica (al ligamento de Cooper).

El tratamiento del prolapso de cúpula vaginal que empleamos sistemáticamente sigue la técnica propuesta por el Dr. Héctor $E$. Bernal en su extraordinario trabajo sobre el tema y publicado en la revista de la Sociedad en el Vol. XI, No. 3, correspondiente a julio-agosto de 1960. Nosotros no vacilamos en recomendarlo y a pesar de que en esta revisión únicamente aparecen tres casos, nos comprometemos a presentar en próxima oportunidad una más cuidadosa y amplia revisión que nos permita mostrar todos los casos operados en el Hospital de la Samaritana desde hace más de 20 años, cuando aprendimos la técnica del propio Dr. Bernal. 
Esta técnica la hemos practicado y enseñado a todos nuestros residentes obteniendo resultados ampliamente satisfactorios en la corrección de esta clase de prolapso. Básicamente, como lo describe el propio Dr. Bernal en el mencionado trabajo, comprende tres partes:

“I - Disección muy amplia del perineo con desprendimiento de la pared posterior de la vagina hasta la propia cúpula vaginal, en cuyo trayecto se ponen al descubierto los músculos perineales superficiales y profundos, la cara anterior del recto y el enterocele cuyo saco una vez aislado, reducido el contenido y ligado, se secciona y se fija a los haces profundos de los pilares del elevador.

II - Cura del cistocele en la forma habitual, pero permitiendo la formación, a expensas de una porción de mucosa vaginal que como tejido heterotópico queda incluido profundamente, de un tubo que a manera de arco de cúpula contribuye a sostener la bóveda vaginal.

III - Perineorrafia muy amplia que incluye parcialmente los músculos superficiales del periné".

\section{MATERIAL Y METODOS}

a. Se seleccionaron las historias clínicas con diagnóstico de prolapso genital, desde mayo de 1979 hasta mayo 31 de 1984 y se extrajeron datos de edad, paridad, grado de prolapso genital, intervención quirúrgica practicada, complicaciones intra y post-operatorias, estadía intrahospitalaria post-operatoria, sintomatología, recidivas.

b. Se distribuyeron las pacientes en grupos de edad y se hicieron cruces de las siguientes variables: edad y grado de prolapso, paridad y grado de prolapso, sintomatología y grado de prolapso, corrección practicada, resultado y recidi- va, último parto y tiempo de aparición del prolapso.

c. Se elaboraron cuadros y gráficas representativas de los datos obtenidos en los cruces anteriores y se analizaron.

\section{RESULTADOS}

A. Características de la muestra estudiada.

B. Tratamiento del prolapso genital en la muestra estudiada.

C. Resultados del tratamiento empleado.

\section{Tipo de prolapso (Cuadro No. 1)}

El hallazgo más frecuente en el trabajo fue el prolapso grado II (50.4\%), seguido del III (46.7\%).

\section{Cuadro No. 1}

INCIDENCIA DE CADA TIPO DE PROLAPSO

\begin{tabular}{|l|c|}
\hline Tipo Prolapso & $\%$ \\
\hline II sin elongation colli & 39 \\
II con elongation colli & 11,4 \\
III & 46,7 \\
Cupula & 3 \\
\hline \multicolumn{2}{|c|}{$100 \%$} \\
No. $=247$
\end{tabular}

Estos datos están influidos por factores determinantes tales como la desnutrición y la atención de partos por empíricas, situaciones muy frecuentes en nuestra clientela hospitalaria.

\section{Edad (Cuadro No. 2).}

El promedio de edad fue de 55 años, época en la cual las pacientes en su mayoría han entrado en la menopausia 
Cuadro No. 2

CARACTERISTICAS DE LA MUESTRA EDAD

Edad Promedio $=55$ años

EDAD SEGUN TIPO DE PROLAPSO

\begin{tabular}{|c|c|}
\hline Tipo Prolapso & Edad $\overline{\mathrm{X}}$ (años) \\
\hline II sin elongation colli & 51 \\
II con elongation colli & 39 \\
III & 58 \\
Cupula & 64 \\
\hline
\end{tabular}

No. $=247$

y han terminado los eventos reproductivos. El hipoestrogenismo y la paridad nos explican, entonces, este hallazgo.

Si analizamos la edad en función del grado de prolapso genital veremos que a mayor edad, mayor grado de prolapso, lo cual está acorde con los informes de la literatura mundial y nos confirma una vez más la influencia de edad e hipoestrogenismo en la etiología del prolapso genital.

\section{Paridad (Cuadro No. 3)}

El promedio total fue de 7 hijos, lo cual nos destaca la relación entre paridad y prolapso genital, a la vez que nos comprueba el papel de los partos en la génesis del prolapso genital.

Si analizamos la paridad según grado de prolapso, vemos que a mayor paridad corresponde un tipo más avanzado de prolapso genital, lo cual está explicado por el hecho de ser los partos, en especial aquellos sin atención médica, muy frecuentes, prolongados o rápidos, un factor determinante del relajamiento de las estructuras pélvicas. No hubo casos de prolapso en nulíparas.
Cuadro No. 3

CARACTERISTICAS DE LA MUESTRA PARIDAD

Paridad promedio $=7$ hijos

PARIDAD SEGUN TIPO DE PROLAPSO

\begin{tabular}{|l|c|}
\hline Tipo Prolapso & Paridad $\overline{\mathbf{X}}$ (hijos) \\
\hline II sin elongation colli & 6,7 \\
II con elongation colli & 6,0 \\
III & 8,0 \\
Cupula & 7,6 \\
\hline \multicolumn{2}{|c|}{ No. $=247$}
\end{tabular}

\section{Relación con IUE (Cuadro No. 4)}

En total, aproximadamente la mitad de los casos se asoció a IUE. De acuerdo

Cuadro No. 4

INCIDENCIA TOTAL DE I. U. E.

\begin{tabular}{|l|l|}
\hline Tipo & $\%$ \\
\hline Prolapsos asociados a I.U.E. & 44 \\
Prolapsos sin I.U.E. & 56 \\
\hline
\end{tabular}

No. $=247100 \%$

Cuadro No. 5

ASOCIACION CON I.U.E. SEGUN TIPO DE PROLAPSO

\begin{tabular}{|c|c|c|}
\hline \multirow{2}{*}{ Tipo Prolapso } & \multicolumn{2}{|c|}{$\begin{array}{c}\text { Asociación con } \\
\text { I.U.E. }\end{array}$} \\
\cline { 2 - 3 } & \multicolumn{1}{|c|}{ Si } & \multicolumn{1}{|c|}{ No } \\
\hline II sin elongation colli & 72,4 & 27,6 \\
II con elongation colli & 53 & 47 \\
III & 28,5 & 71,5 \\
Cupula & 0 & 100 \\
\hline
\end{tabular}

No. $=247$ 
al tipo de prolapso, el 71.5\% de prolapsos III no presentó este síntoma, situación que se explicaría por la severa distorsión de los ángulos útero-vesicales y del peso pélvico, que se presentan en el prolapso completo. Aquí no se presenta IUE, la tendencia es de una mayor frecuencia de retención urinaria. Este hallazgo coincide con lo informado en la bibliografía.

\section{Síntomas (Cuadros No. 6 y 7 )}

La mitad de las pacientes se quejaron de sensación de cuerpo extraño en la

Cuadro No. 6

SINTOMATOLOGIA

Síntomas más frecuentes

\begin{tabular}{|l|l|}
\hline Sintoma & $\circ$ \\
\hline Sensación cuerpo extraño & 50 \\
I. U. E. Exclusivamente & 0 \\
I. U. E. + sensación & \\
cuerpo extraño & 48 \\
Retención urinaria & 2 \\
\hline
\end{tabular}

$100 \%$

No. $=247$

Cuadro No. 7

SINTOMATOLOGIA SEGUN TIPO DE PROLAPSO

\begin{tabular}{|c|l|c|}
\hline $\begin{array}{c}\text { Tipo } \\
\text { Prolapso }\end{array}$ & \multicolumn{1}{|c|}{ Sintomas } & \% \\
\hline \multirow{2}{*}{ II } & Sensación cuerpo extraño & 27,6 \\
& Cuerpo extraño + I.U.E. & 72,4 \\
\hline Elongation & Sensación cuerpo extraño & 47,0 \\
Colli & Cuerpo extraño + I.U.E. & 53,0 \\
\hline \multirow{2}{*}{ Cupula } & Sensación cuerpo extraño & 67,0 \\
& Retención urinaria & 33,0 \\
\hline \multirow{3}{*}{ III } & Sensación cuerpo extraño & 28,5 \\
& Cuerpo extraño + I.U.E. & 66,0 \\
& Retención urinaria & 5,5 \\
\hline
\end{tabular}

No. $=247$ vagina y la mitad restante de IUE asociada a sensación de cuerpo extraño. Solamente dos casos manifestaron únicamente retención urinaria, que correspondieron a prolapso de cúpula, situación en que se podrán también aplicar las explicaciones anotadas en el párrafo anterior.

\section{Duración de la Enfermedad (Cua- dro No. 8)}

Fue en promedio 4,75 años antes de la consulta donde se detectó la enfermedad. Esto nos muestra la renuencia de nuestros enfermos a acudir a la consulta de ginecología, tal vez por un falso pudor o por ignorancia franca.

Cuadro No. 8

\section{DURACION DE LA ENFERMEDAD}

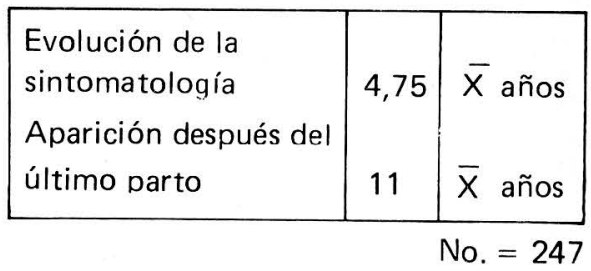

7. Aparición después del último Parto. (Cuadro No. 8).

La sintomatología del prolapso apareció en promedio, 11 años después del último parto, lo cual sugiere que el prolapso no se presenta de inmediato después del parto, sino muchos años después. Tal vez por requerirse de tiempo para que las estructuras pélvicas presenten relajamiento completo o porque quizá se requiera la aparición de vejez con sus cambios atróficos del tejido conectivo y su hipoestrogenismo, para que se manifieste el prolapso genital.

\section{Pautas de Manejo (Cuadro No. 9).}

Se ilustran aquí las técnicas quirúrgicas que empleamos en el Hospital para 
Cuadro No. 9

PAUTAS

\begin{tabular}{|l|l|c|}
\hline \multicolumn{1}{|c|}{ Diagnóstico } & \multicolumn{1}{|c|}{ Tratamiento } & $\begin{array}{c}\text { Cumplimiento } \\
\text { en la muestra \% }\end{array}$ \\
\hline $\begin{array}{l}\text { Prolapso II } \\
\begin{array}{l}\text { Prolapso II elongatio } \\
\text { colli, mujer jóven }\end{array}\end{array}$ & $\begin{array}{l}\text { Kelly clásico o Kelly modif. } \\
\text { Manchester }\end{array}$ & $93 \%$ \\
$\begin{array}{l}\text { Prolapso III } \\
\begin{array}{l}\text { Prolapso cupula post. } \\
\text { Histerect. vaginal }\end{array}\end{array}$ & Histerect. vaginal + C. Celes & $95 \%$ \\
\hline
\end{tabular}

No. $=247$

el tratamiento de los diferentes tipos de prolapso. Se anotan también el porcentaje de cumplimiento de la pauta anotada. En los casos de prolapso II y de Elongatio Colli hubo algunas pacientes $(7 \% \mathrm{y}$ 5 \% respectivamente) en que no se cumplió la norma, por acuerdo mutuo entre el cirujano y la paciente en el momento inmediatamente anterior a la iniciación del acto quirúrgico.

\section{Incidencia de Cirugías (Cuadro No. 10)}

La cirugía más frecuente fue la histerectomía vaginal (58\% de los casos), seguida de la operación de Kelly Clásico (25\%); las menos frecuentes fueron el Le Fort y la corrección de prolapso de de cúpula vaginal. Estos datos se correlacionan con la incidencia de los diferentes tipos de prolapso (Cuadro No. 1) y con el manejo para cada uno de éstos (Cuadro No. 9).

10. Descripción general de las complicaciones (Cuadro No. 11).

La mayoría de las complicaciones presentadas fue de tipo post-operatorio (48\%) y una minoría (7.4\%) de tipo intraoperatorio, lo cual se explica por
Cuadro No. 10

INCIDENCIA DIFERENTES CIRUGIAS

\begin{tabular}{|l|r|}
\hline Cirugía & \multicolumn{1}{|c|}{$\%$} \\
\hline Kelly clásico & 25,0 \\
Kelly modificado & 4,5 \\
Histerect. vaginal & 5,8 \\
Corrección vaginal de & 3,0 \\
prolapso cupula & 7,0 \\
Manchester & 2,5 \\
Lefort & $100 \%$ \\
\hline
\end{tabular}

No. $=247$

Cuadro No. 11

MORBILIDAD

\begin{tabular}{|l|c|}
\hline Tipo de morbilidad & $\%$ \\
\hline Complicaciones intraoperatorias & 7,4 \\
Complicaciones del & \\
post operatorio inmediato & 48,0 \\
Morbilidad Total & 55,4 \\
\hline
\end{tabular}


considerarse a la retención urinaria postoperatoria como complicación; además de que la correcta aplicación de las técnicas quirúrgicas y la detección y corrección inmediatas de fallas durante la cirugía reducen significativamente la morbilidad intraoperatoria.

11. Entidades que constituyen las complicaciones intra-operatorias (Cuadro No. 12).

Dentro de las complicaciones intraoperatorias la más frecuente fue el sangrado profuso $(77 \%$ de las cirugías complicadas intraoperatoriamente), hecho explicado por la generosa irrigación de la mucosa vaginal y de la fascia pubovesical-uterina. Este dato coincide con otros trabajos publicados en la literatura, que califican a la hemorragia como la primera complicación post-operatoria. Vale la pena aclarar que aquí se considera como hemorragia intra-operatoria profusa a aquella que requirió transfusión sanguínea de 500 c.c. o más para corregirla.

Cuadro No. 12

CONDICIONES PATOLOGICAS INTRAOPERATORIAS DETECTADAS

\begin{tabular}{|l|c|}
\hline Situación patológica & do \\
\hline Sangrado profuso & 77,0 \\
Lesión pared rectal ant. & $14, \mathrm{C}$ \\
Perforación vejiga urinaria & 9,0 \\
Morbilidad intraoperatoria total & 7,4 \\
\hline
\end{tabular}

No. $=247$

La segunda complicación fue la ruptura de recto $(14 \%$ de las complicaciones), que se presenta en algunos casos por adherencias marcadas entre mucosa vaginal y fascia rectovaginal con lesión severa de periné.
12. Complicaciones intra-operatorias en cada tipo de Cirugía. (Cuadro No. 13).

El tipo de cirugía que más complicaciones intra-operatorias presentó fue la histerectomía vaginal, morbilidad del $11 \%$, seguida de la operación de Manches-

Cuadro No. 13

MORBILIDAD INTRAOPERATORIA SEGUN TIPO DE CIRUGIA

\begin{tabular}{|l|c|r|}
\hline \multicolumn{1}{|c|}{ Tipo de cirugra } & $\begin{array}{c}\text { Morbilidad } \\
\%\end{array}$ & N \\
\hline Histerectomia vaginal & 11 & 27 \\
Manchester & 3 & 7 \\
Kelly modificado & 0,4 & 1 \\
Kelly clásico & 0 & 0 \\
Corr. Prolapso cupula & 0 & 0 \\
Lefort & 0 & 0 \\
& & 35 \\
\hline
\end{tabular}

No. $=247$

Cuadro No. 13A

\begin{tabular}{|c|c|c|c|}
\hline Cirugra & Complicación & $\mathbf{N}$ & 010 \\
\hline Kelly & - & 0 & 0 \\
\hline $\begin{array}{l}\text { Kelly } \\
\text { modificado }\end{array}$ & sangrado & 1 & 2,8 \\
\hline $\begin{array}{l}\text { Histerectomia } \\
\text { V. }\end{array}$ & $\begin{array}{l}\text { sangrado } \\
\text { lesión vejiga } \\
\text { lesión recto }\end{array}$ & $\begin{array}{r}23 \\
1 \\
3\end{array}$ & $\begin{array}{r}65,7 \\
2,8 \\
8,5\end{array}$ \\
\hline Manchester & $\begin{array}{l}\text { sangrado } \\
\text { lesión vejiga } \\
\text { lesión recto }\end{array}$ & $\begin{array}{l}3 \\
2 \\
2\end{array}$ & $\begin{array}{l}8,5 \\
5,6 \\
5,6\end{array}$ \\
\hline Cupula & - & 0 & 0 \\
\hline Lefort & - & 0 & 0 \\
\hline
\end{tabular}


ter (3). Estos hallazgos coinciden con lo expresado en la literatura mundial y se explican por ser estos tipos de cirugía los más extensos dentro de los usados para la corrección del prolapso genital. El Cuadro 13A muestra las entidades nosológicas que se constituyeron en complicaciones intraoperatorias en cada una de las modalidades quirúrgicas empleadas; se confirma de nuevo el primer lugar para el sangrado.

\section{Entidades que constituyen las com-} plicaciones post-operatorias (Cuadro No. 14).

La complicación más frecuente fue la retención urinaria $(51.4 \%)$. Se discute si la retención urinaria es realmente una complicación o si al contrario, como afirman algunos autores, es un síntoma que indica una corrección adecuada de la incontinencia urinaria. La segunda complicación fue la infección urinaria (28.4을, explicable por el uso de la sonda vesical post-operatoria.

Cuadro No. 14

\section{MORBILIDAD POST-OPERATORIA \\ CONDICIONES PATOLOGICAS POST-..OPERATORIAS DETECTADAS}

\begin{tabular}{|l|c|r|}
\hline Condición Patológica & Morbilidad \% & N \\
\hline Retención urinaria & 51,4 & 63 \\
Infección urinaria & 28,4 & 35 \\
Infección local & 15,4 & 19 \\
Pat. psiquiatrica & 1,6 & 2 \\
Bridas vaginales & 0,8 & 1 \\
Ligadura ureteres & 0,8 & 1 \\
llio metabólico & 0,8 & 1 \\
Hematoma mucosa V. & 0,8 & 1 \\
\hline Total & 48,0 & \\
\hline
\end{tabular}

No. $=123$
Estos datos y los que corresponden a las demás complicaciones post-operatorias no difieren de los informados en la bibliografía.

\section{Complicaciones post-operatorias en cada tipo de cirugía (Cuadros Nos. 15 y $15 \mathrm{~A}$.$) .$}

La cirugía que más complicaciones post-operatorias presentó fue la histerectomía vaginal (25.5\%), seguida de la operación de Kelly clásico. Estos hallazgos son similares a los descritos en otros trabajos y como ya lo expresamos, se deben a la corrección misma de la IUE, que puede ocasionar retención urinaria temporal y al uso de la sonda vesical, que se hace obligatorio en este tipo de post-operatorio y que potencialmente genera infección urinaria. En el Cuadro No. 15A se muestra en forma discriminada para cada cirugía, las condiciones patológicas que constituyeron la complicación. De nuevo, la retención urinaria fue la más frecuente.

Cuadro No. 15

MORBILIDAD POST-OPERATORIA SEGUN TIPO DE CIRUGIA (\% Total de Cirugia)

\begin{tabular}{|l|c|r|}
\hline Cirugía & $\begin{array}{c}\text { Morbilidad } \\
\text { olo }\end{array}$ & N \\
\hline Kelly & 15,7 & 39 \\
Kelly modificado & 2,0 & 5 \\
Histerectomia vaginal & 25,1 & 62 \\
Manchester & 6,8 & 17 \\
Lefort & 0 & 0 \\
Cupula & 0,8 & 2 \\
& \multicolumn{2}{|c|}{ No. $=247$} \\
\hline \multicolumn{2}{|c|}{} \\
\hline
\end{tabular}


Cuadro No. $15 \mathrm{~A}$

MORBILIDAD POST -OPERATORIA SEGUN TIPO DE CIRUGIA

\begin{tabular}{|c|c|c|c|}
\hline Cirugra & Complicación & $\mathbf{N}$ & 010 \\
\hline Kelly & $\begin{array}{l}\text { Retención } \\
\text { Inf. urinaria } \\
\text { Bridas }\end{array}$ & $\begin{array}{r}22 \\
16 \\
1\end{array}$ & $\begin{array}{r}19,0 \\
13,0 \\
0,8\end{array}$ \\
\hline $\begin{array}{c}\text { Kelly } \\
\text { Modificado }\end{array}$ & $\begin{array}{l}\text { Inf. urinaria } \\
\text { Retención } \\
\text { Inf. local } \\
\text { Psiquiatricas }\end{array}$ & $\begin{array}{l}2 \\
1 \\
1 \\
1\end{array}$ & $\begin{array}{l}1,6 \\
0,8 \\
0,8 \\
0,8\end{array}$ \\
\hline $\begin{array}{l}\text { Histerec- } \\
\text { tomia V. }\end{array}$ & $\begin{array}{l}\text { Retención } \\
\text { Absceso Cupula } \\
\text { Inf. urinaria } \\
\text { Obst. ureteres } \\
\text { IUO } \\
\text { Psiquiatricas }\end{array}$ & $\begin{array}{r}32 \\
14 \\
13 \\
1 \\
1 \\
1\end{array}$ & $\begin{array}{r}26 \\
11,0 \\
10,0 \\
0,8 \\
0,8 \\
0,8\end{array}$ \\
\hline Manchester & $\begin{array}{l}\text { Retención } \\
\text { Inf. local } \\
\text { Inf. urinaria } \\
\text { Hematoma }\end{array}$ & $\begin{array}{l}6 \\
4 \\
1 \\
1\end{array}$ & $\begin{array}{l}4,8 \\
3,2 \\
0,8 \\
0,8\end{array}$ \\
\hline Cupula & Retención & 2 & 1,6 \\
\hline Lefort & - & 0 & 0 \\
\hline
\end{tabular}

15. Estancia post-operatoria (Cuadro No. 16).

En promedio el número de días estancia fue de 8 días, en términos generales, que se aumentarán en más o menos 3 días cuando hubo morbilidad intraoperatoria. La cirugía cuyo post-operatorio ocupó mayor número de días estancia fue la histerectomía vaginal seguida por el Kelly modificado. En el caso de la histerectomía, esto se explica por ser la técnica quirúrgica más extensamente utilizada y en el caso del Kelly modificado, por el tipo de puntos que se trazan para corregir la incontinencia,
Cuadro No. 16

\begin{tabular}{|c|c|c|c|c|}
\hline \multirow[b]{2}{*}{ Tipo Cirugia } & \multicolumn{4}{|c|}{7 DIAS } \\
\hline & $\begin{array}{l}\text { Sin com- } \\
\text { plicación }\end{array}$ & $\begin{array}{l}\text { Compli- } \\
\text { cacion Intra }\end{array}$ & $\begin{array}{l}\text { Compli- } \\
\text { cación Post. }\end{array}$ & Total \\
\hline Kelly Clásico & 5,8 & - & 9,2 & 7,5 \\
\hline Kelly modificado & 6,6 & 12,0 & 12,0 & 9,0 \\
\hline Histerectomia vaginal & 7,1 & 14,0 & 13,0 & 11,3 \\
\hline Manchester & 6,6 & 7,0 & 9,0 & 7,5 \\
\hline Lefort & 6,0 & - & 9,0 & 7,5 \\
\hline $\begin{array}{l}\text { Correción vaginal de } \\
\text { prolapso de cupula }\end{array}$ & 5,0 & - & 8,0 & 6,5 \\
\hline \multicolumn{4}{|l|}{$N=247$} & 8,1 \\
\hline $\bar{X}$ días estancia post-op & \multicolumn{4}{|c|}{$\begin{array}{l}\text { Según tipo de cirugía y según } \\
\text { complicaciones presentadas }\end{array}$} \\
\hline
\end{tabular}

con los cuales cabe esperar retención urinaria más frecuente.

\section{Hallazgos de Patología (Cuadros Nos. 17 y 17A).}

Se ilustran aquí los hallazgos de patología, siendo los más frecuentes los cambios por prolapso (48\%) y cervicitis crónica $(17 \%)$. Esto se explica por las características mismas de la entidad que estamos tratando. Los hallazgos descritos en este cuadro no difieren de lo descrito en la literatura mundial, a excepción de 6 casos de displasia leve y uno de displa-

Cuadro No. 17

HALLAZGOS DE ANATOMIA PATOLOGICA

\begin{tabular}{|l|l|r|}
\hline \multicolumn{1}{|c|}{$\begin{array}{c}\text { Diagnóstico anatomo- } \\
\text { patológico }\end{array}$} & $\%$ & N \\
\hline Cambios por Prolapso & 48 & 96 \\
Cervicitis crónica & 16,5 & 32 \\
Miomatosis uterina & 8,0 & 15 \\
submucosa & 7,7 & 14 \\
Acantosis exócervical & 5 & 9 \\
Endometrio atrofico & 3 & 6 \\
Polipo endometrial & & \\
Metaplasia escamosa & 3 & 6 \\
endocervical &
\end{tabular}


Cuadro No. 17A

HALLAZGOS DE ANATOMIA PATOLOGICA

\begin{tabular}{|l|c|c|}
\hline \multicolumn{1}{|c|}{$\begin{array}{c}\text { Diagnóstico anatomo- } \\
\text { patológico }\end{array}$} & 이 & N \\
\hline $\begin{array}{l}\text { Displasia leve exocervical } \\
\text { Hiperplasia glanduloquís- }\end{array}$ & 2,5 & 5 \\
tica endometrio & 2,5 & 5 \\
Endometrio proliferativo & 1,5 & 3 \\
Displasia moderada & & \\
exocervix & 0,5 & 1 \\
Ca. cervix infiltrante & 0,5 & 1 \\
Adenomiosis & 0,5 & 1 \\
\hline $\mathrm{N}=192$ & $100 \%$
\end{tabular}

sia moderada. Además de un caso de Ca. de cervix infiltrante que no fue detectado pre-operatoriamente, debido a que la citología fue informada como un Pap. II y los hallazgos clínicos no permitieron sospecharlo. Estos hallazgos de lesiones pre-malignas o malignas del cervix son extremadamente raros en asocio con el prolapso y no tenemos explicación satisfactoria para este hallazgo. Se destaca que estas neoplasias del cervix se encontraron todas en casos de prolapso genital III.

\section{Hallazgos de Citología (Cuadro No. 18).}

El 93 de las citologías fueron negativas para tumor, lo cual está de acuerdo con lo expresado en diferentes trabajos que aparecen en la bibliografía del final del presente estudio.

7응 de las citologías fueron anormales y correspondieron a los casos de displasia de cervix que fueron descritos en el párrafo anterior, situación muy raramente informada y que nuevamente presentamos aquí sin explicación satisfactoria.
Cuadro No. 18

TIPOS DE CITOLOGIAS

CERVICO-VAGINALES

\begin{tabular}{|c|c|c|}
\hline Clase & $\%$ & \multirow{5}{*}{$\begin{array}{l}\text { Todas en prolapso } \\
\text { genital III }\end{array}$} \\
\hline 1 & 67,6 & \\
\hline 11 & 25,3 & \\
\hline III & 6,1 & \\
\hline IV & 1,0 & \\
\hline
\end{tabular}

\section{Pacientes que acudieron a control Post-operatorio (Cuadro No. 19).}

El $60 \%$ de las pacientes acudieron a algún control ginecológico dentro del año siguiente a su cirugía. El $40 \%$ restante nunca regresó después de su salida del hospital, tal vez por permanecer asintomáticas o por vivir en provincia; no se investigó esta variable en el presente estudio.

Cuadro No. 19

SEGUIMIENTO AL AÑO

POST-OPERATORIO

\begin{tabular}{|l|r|c|}
\cline { 2 - 3 } \multicolumn{1}{c|}{} & N & $\%$ \\
\hline Regresaron & 148 & 59 \\
No regresaron & 99 & 41 \\
Total & 247 & $100 \%$ \\
\hline
\end{tabular}

19. Síntomas que reaparecieron luego del tratamiento quirúrgico (Cuadro No. 20).

El síntoma que más frecuentemente recidivó fue la incontinencia urinaria de esfuerzo; raramente reaparecieron los celes o el prolapso de la cúpula vaginal. Quizás esto se debió a error en la técnica quirúrgica, infección post-operatoria o debilidad persistente del tejido correc- 
tivo, que comprometería en forma persistente el resultado de la cirugía.

Cuadro No. 20

\section{SINTOMAS QUE REAPARECIERON} LUEGO DE LA CIRUGIA

\begin{tabular}{|c|c|c|}
\hline Tipo de Recidiva & $\%$ & $\mathbf{N}$ \\
\hline $\begin{array}{l}\text { Reaparición de IUE } \\
\text { exclusivamente }\end{array}$ & 47 & 16 \\
\hline Reaparición de celes & & \\
\hline $\begin{array}{l}\text { sintomático } \\
\text { Reaparición celes }\end{array}$ & 35 & 12 \\
\hline sintomáticos + IUE & 9 & 3 \\
\hline Prolapso cupula & 9 & 3 \\
\hline$N=34$ & 100 & 34 \\
\hline
\end{tabular}

20. Exitos y fracasos del tratamiento quirúrgico (Cuadro No. 21).

Se obtuvo un 23 \% de recidivas frente a un $77 \%$ de curaciones totales de la sintomatología. Estos valores de éxito terapéutico son superiores a los informados en la literatura, que oscilan en un $60-65 \%$ de curaciones. La cirugía que más recidivas presentó fue el Kelly modificado, técnica que en un comienzo se acogió en el Servicio con gran entusiasmo pero que, viéndose su alto índice de fracasos (45\%), se abandonó en 1981, luego de 11 intervenciones. Le siguen el Kelly clásico con 28 o de fracasos y la histerectomía vaginal con $19 \%$

\section{Recidivas presentadas en cada tipo de Cirugía (Cuadro No. 22).}

El Cuadro No. 22 nos muestra los diferentes tipos de recidivas presentados en cada modalidad quirúrgica. Se nota de nuevo una tendencia a sobresalir la IUE como recidiva más frecuente, acorde con lo descrito en la bibliografía y en el Cuadro No. 20.

Cuadro No. 21

RECIDIVAS SEGUN TIPO DE CIRUGIA

\begin{tabular}{|l|r|r|r|r|r|r|}
\cline { 2 - 7 } \multicolumn{1}{c|}{} & \multicolumn{2}{c|}{ Cirugías } & \multicolumn{2}{c|}{ Fracasos } & \multicolumn{2}{c|}{ Curaciones } \\
\hline \multicolumn{1}{c|}{ Tipo cirugía } & $N$ & \multicolumn{1}{c|}{$\%$} & $N$ & $\%$ & $N$ & $\%$ \\
\hline $\begin{array}{l}\text { Kelly clásico } \\
\text { Kelly modificado }\end{array}$ & 11 & 25,6 & 11 & 28 & 27 & 72 \\
$\begin{array}{l}\text { Histerectomia } \\
\text { vaginal }\end{array}$ & 76 & 51,3 & 15 & 19 & 61 & 81 \\
$\begin{array}{l}\text { Corrección vaginal } \\
\text { prolapso C. }\end{array}$ & 3 & 2,0 & 0 & 0 & 3 & 100 \\
Manchester & 17 & 11,4 & 3 & 17 & 14 & 83 \\
Lefort & 3 & 2,0 & 0 & 0 & 3 & 100 \\
\hline \multicolumn{1}{|c|}{ Totales } & 148 & 100 & 34 & 23 & 114 & 77 \\
\hline
\end{tabular}


Cuadro No. 22

RECIDIVAS SEGUN TIPO DE CIRUGIA

\begin{tabular}{|l|l|r|r|}
\hline \multicolumn{1}{|c|}{ Cirugía } & \multicolumn{1}{c|}{ Recidiva } & N & \% \\
\hline Kelly & IVE & 4 & 12 \\
modificado & celes más IVE & 1 & 3 \\
\hline \multirow{4}{*}{ Kelly } & Celes & 5 & 15 \\
& IVE & 5 & 15 \\
& celes mas IVE & 1 & 3 \\
\hline \multirow{3}{*}{ Histerectomia } & Celes & 5 & 15 \\
V. & IVE & 6 & 18 \\
& Cupula & 3 & 9 \\
& Celes más IVE & 1 & 3 \\
\hline \multirow{3}{*}{ Manchester } & Celes & 2 & 6 \\
\hline Cupula & IVE & 1 & 3 \\
\hline Lefor & - & - & - \\
\hline
\end{tabular}

\section{RESUMEN Y CONCLUSIONES}

Encontramos al analizar la incidencia de los diferentes tipos de prolapso, la edad, paridad, asociación con I.U.E. Y síntomas que en más de la mitad de los casos corresponden a prolapso grado II (50.4\%); de ellos los que cursaron sin Elongation Colli constituian el 39\%, tenían más de 51 años y eran grandes multíparas, con 7 partos en promedio y el síntoma predominante era la sensación de cuerpo extraño asociado a I.U.E. en el $72.4 \%$

En el prolapso grado III persiste la sensación de cuerpo extraño en un alto porcentaje, $66 \%$, pero disminuye la I.U.E. a un $28.5 \%$, siempre en grandes multíparas, promedio de 8 y en edad postmenopáusica, 58 años de promedio. Consideramos que indudablemente la multiparidad asociada a las fallas tróficas que ocasiona el hipoestrogenismo de la post- menopausia juega papel importante en las fallas del soporte genitourinario.

Se practicó histerectomía vaginal como la técnica más frecuente para tratar el prolapso genital, 58\%. Esto está de acuerdo con lo analizado por los diferentes autores del tema. En todas las cirugías la morbilidad intra-operatoria fue del 7.4\%; en su mayor parte, $77 \%$ constituido por el sangrado profuso. Debemos entender que la mayoría de los casos son practicados por personal en entrenamiento, además del hecho de que los plexos venosos de la región favorecen esta situación.

Las complicaciones pcst-operatorias constituyeron el $48 \%$, siendo la retención urinaria la mayor causa, $51.4 \%$, por encima de la infección.

La estancia post-operatoria la consideramos realmente alta y guarda relación directa con las complicaciones intra y post-operatorias, dando el mayor promedio la histerectomía vaginal. Como explicación tenemos que dadas las condiciones socio-económicas de nuestras pacientes hospitalarias, la retención urinaria que podría ser manejada ambulatoriamente, la tratamos en forma intrahospitalaria.

El $82 \%$ de la patología encontrada al estudio A.P. fue benigna, constituida por cambios propios del prolapso en un 48\%; cervicitis crónica en un $16.5 \%$ y miomatosis uterina un $8 \%$. Esto está de acuerdo ćon lo ordinariamente informado. Sin embargo, se encontraron en 6 casos de prolapso III asociación con displasia leve y moderada y un caso más informado después del estudio A.P. de la pieza quirúrgica como C.A. infiltrante de cervix, lo cual no es frecuente verlo informado.

El seguimiento post-operatorio fue inadecuado, consiguiéndose únicamente 
que el $59 \%$ de las pacientes regresaron cuando menos una vez después del egreso hospitalario.

La persistencia de I.U.E. se observó en el $47 \%$ de las pacientes que regresaron al control, siendo de suponer que es bastante más alto. Esto es explicable dado el concepto actualmente más aceptado de que las técnicas para la corrección del prolapso uretrovesical por vía vaginal no llenan los requisitos para la cura de la I.U.E.

El porcentaje de curación obtenido con el tratamiento quirúrgico del prolapso fue superior al $72 \%$ a excepción de los casos en los cuales se empleó la operación de Kelly modificada donde se encontró un fracaso del 45\%, dado especialmente por la persistencia del síntoma de I.U.E. ya explicado.

\section{EPIDEMIOLOGY OF GENITAL PROLAPSE}

\section{SUMMARY}

The authors analyzed the incidence of the diverse types of prolapse, the age, parity, association with stress urinary incontinence, and symptoms that in more than $50 \%$ of the cases correspond to prolapse II (50.4\%); of these, 39\% did not develop Ellongatio colli, were more than 51 years old, grand multiparas with an average of 7 deliveries and the predominant symptom being the sensation of a foreign object associated with S.U.I. in $72.4 \%$ of the cases.

In patients with prolapse III, the sensation of a foreign object persists in $66 \%$ of the cases, while S.U.I. decreases to $28.5 \%$, always in grand multipara, average of 8 deliveries and 58 years old in average. Undoubtedly, multiparity associated with trophic effects of hypoestrogen in the post-menopause period, plays a very important role in faulty genito-urinary support.

58 \% of the prolapses were treated by vaginal hysterectomy, which is a widespread technique. Intra-operatory morbidity was $7.4 \%$, of which $77 \%$ were cases of severe bleeding. It must be understood that most surgeries are conducted by in-service trainees, besides the blood vessel factor that makes that area particularly vulnerable.

Post-operatory complications were reported in $48 \%$ of the cases, urinary retention representing the highest incidence, $51.4 \%$ above infection.

The number of hospital days was very high and is directly related with intra and post-operatory complications, especially in the cases of vaginal hysterectomy. This is due probably to the fact that the socio-economic level of the patients make ambulatory treatment of urinary retention practically impossible, or unwise.

$82 \%$ of the pathology found after the anatomo-pathological tests was benign and in $48 \%$ of the cases due to normal prolapse changes; $16.5 \%$ were due to chronic cervicitis, and $8 \%$ to uterine miomatosis. These findings are similar to those ordinarily found. Nevertheless,in three cases of prolapse III the tests reported mild and moderate displasia, and one more case informed after the A.P. as infiltrating cervicel cancer.

Post-operatory follow-up was not excellent, as only $59 \%$ of the women 
returned at least once for follow-up check-ups.

Persistence of S.U.I. was found in $47 \%$ of the women that returned for followup, but authors believe the incedence to be much higher. This could be explained by the fact that the techniques used to

\section{BIBLIOGRAFIA}

BECERRA J., NAVARRO, B. Manejo Quirúrgico del Prolapso Genital. Trabajo de Promoción de R II - R III, Hospital Universitario La Samaritana, octubre de 1983.

BENSON, R. Relajaciones de los Soportes Pélvicos. Diagnóstico y Tratamiento GinecoObstétricos. Cap. 11, Págs. 250-267, Manual Moderno, 1979.

BERNAL, H.E. El Prolapso de Cúpula Vaginal. Revista Colombiana de Obstetricia y Ginecología. Vol. XI, No. 3, Pág. 394.

GORDON, BEMIO G. Reparación de Enterocele (Hernia de la Pared Vaginal Posterior). Clínicas Obstétricas y Ginecológicas. Septiembre de 1975, Págs. 3-19 Interamericana.

LOMANTO, A. Incontinencia urinaria de esfuerzo en la mujer. Tratamiento. Primeras Jornadas de Ginecología y Obstetricia. Clinica Hospital Fray Bartolomé de las Casas, P. 113, mayo de 1984.

J. GONZALEZ, Merlo. Prolapso Genital. Ginecología. Cap. 2, Salvat, 1983. Págs. 47-51.

KASER, O. FRIEDBERG, V. Anomalías de Posición. Ginecología y Obstetricia. Gine- correct uretrovesical prolapse through the vagina do not cure S.U.I.

Surgical treatment of prolapses proved successful in more than $72 \%$ of the cases, but failed in $45 \%$ of the cases treated with the modified Kelly operation, because of the persistence of S.U.I. already described.

cología Especial III. Cap. 6, Págs. 696-753, Salvat, 1974.

KERN, G. Sistema de Sustentación del Utero y Suelo de la Pelvis. Anomalías de Posición. Ginecología. Págs. 470-490, Salvat, 1976.

LINARES, J. SUAREZ, D. Manejo de Sondas Vesicales y Mechas Vaginales en Cirugía Ginecológica Vaginal. Trabajo de Promoción R I - R II - Hospital Universitario de la Samaritana, octubre 1983.

RIDLEY, John. Histerectomía Vaginal, Colporrafia Anterior y Posterior, Reparación de Enterocele, Prolapso de Bóveda Vaginal, Cirugía Ginecológica. Capítulo 2, Págs. 3970. In teramericana, 1978.

S.E. JASZCZAK, A. Col. Intrafascial Abdominal \& Vaginal Hysterectomy. Obst. Gynecol. 59: 435,1982

T. LINDE, R. Ginecología Operatoria. Págs. 230-246. Ediciones Científicas, 1956.

URIZA, G. MEDINA, J. Manejo del Prolapso Genital. Revista Colombiana de Obstetricia y Ginecología, Vol. XXXII, No. 3, Págs. 163-175.

WILLIAMS. Obstetricia. Págs. 22-27. Salvat Editores. 